\title{
Brillouin Stokes comb generated in a distributed fiber Raman amplifier
}

\author{
Hugo F. Martins ${ }^{\mathrm{a}, \mathrm{b}}$, Manuel B. Marques ${ }^{\mathrm{a}, \mathrm{b}}$, Orlando Frazão ${ }^{\mathrm{a}}$ \\ ${ }^{a}$ INESC Porto, Rua do Campo Alegre, 687, 4169-007 Porto, Portugal; \\ ${ }^{\mathrm{b}}$ Faculdade de Ciências da Universidade do Porto, Rua do Campo Alegre, 687, 4169-007 Porto, Portugal.
}

\begin{abstract}
A Brillouin Stokes comb laser with increased flatness is reported. The feedback for the laser is provided by a distributed mirror combined with a narrowband seed laser. The Brillouin seed power and wavelength optimization is crucial in order to obtain a uniform power level between Stokes lines. The Brillouin seed must have a relatively large power and its wavelength must be located close to the Raman peak gain region. The flat-amplitude bandwidth is also determined by the choice of Raman pump wavelength. A flat-amplitude bandwidth of $34 \mathrm{~nm}$ from $1538 \mathrm{~nm}$ to $1572 \mathrm{~nm}$ is measured when Raman pump wavelength is set to $1455 \mathrm{~nm} .425$ uniform Brillouin Stokes lines with $0.08 \mathrm{~nm}$ spacing are generated across the wavelength range. The average signal-to-noise ratio of $15 \mathrm{~dB}$ is obtained for all the Brillouin Stokes lines. This type of laser can be used in optical communications as a multiwavelength source and also in metrology as a frequency ruler.
\end{abstract}

Keywords: Non-Linear effects, Distribute mirror, Multiwavelength laser

\section{INTRODUCTION}

The use in optical communication systems is pushing the research in multiwavelength fiber. These lasers can be produced by external channel filtering. The technique is based in slicing a broadband spectrum from a supercontinuum source and has been shown to produce more than 1000 channel optical frequency [1]. Another way to produce these lasers is by internal channel generation, that is using a in-fiber comb filter [2]. In this case a hybrid-gain configuration can be used, using a broadband gain (such as Raman gain) and a narrowband Brillouin gain assisted by Rayleigh scattering, which uses a seed laser at $1550 \mathrm{~nm}$ to generate several lasers in a cascaded process [3].

Different parameters have been tested to improve the quality and number of generated channels. Early studies used erbium gain as the broadband gain [4] but this presented some problems: the gain was limited to a few nanometers which limited the number of generated channels. Raman gain provides a broader band gain and architectures with two, or more, Raman pumps with different wavelengths to increase the gain region have been demonstrated to generate up to 798 channels [5]. The use of cooperative Rayleigh scattering as distributed mirrors in the fiber has also been studied [6]. Forward Raman pumping decreases the threshold to start the Brillouin process [7] but counter-propagating Raman is more efficient and produces less noise in the amplified spectrum [8]. 
Due to the Raman gain properties, a relatively long fiber is required. With the use of dispersion compensating fiber (DCF) the fiber length can be reduced. To increase the amplitude of high order Brillouin Stokes peaks with a few kilometres of fiber, schemes with feedback mechanisms (using discrete mirrors in the end of the fiber) have been suggested to increase the optical signal-to-noise ratio (OSNR) of the peaks [8].

In this paper the dependence of multiwavelength Brillouin/Raman comb fiber laser properties (optical signal-to-noise ratio (OSNR), number of channels and flatness) in wavelength and power of the Brillouin seed and Raman power in a $1 \mathrm{~km}$ DCF fiber are investigated.

\section{EXPERIMENTAL RESULTS}

Figure 1 presents the experimental setup for the Brillouin Raman comb laser. A Raman pump laser at $1455 \mathrm{~nm}$ with a maximum power of $5 \mathrm{~W}$, a tunable seed laser with a maximum power of $11 \mathrm{dBm}$ and two wavelength division multiplexers (WDMs) $(1450 / 1550 \mathrm{~nm})$ are used. A one kilometer dispersion compensating fiber (DCF) with a dispersion coefficient of $-132 \mathrm{ps} / \mathrm{nm} . \mathrm{km}$ is used to create a distributed mirror and Raman gain. An Optical Spectrum Analyzer (OSA) with a maximum resolution of $0.01 \mathrm{~nm}$ was used to observe the optical spectrum.

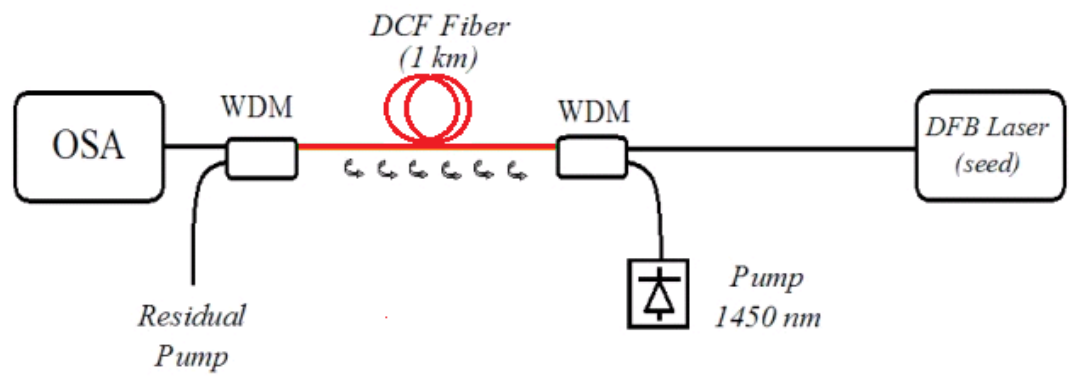

Fig. 1 Experimental setup used to generate a comb laser.

The seed laser is used to start the Brillouin cascaded process. Each new wavelength laser is generated by Brillouin Stokes scattering from the previous laser wavelength, and amplified by Raman gain. Each wavelength laser has a maximum amplitude determined by the stimulated Brillouin threshold, and therefore lasers in the same wavelength region have the same amplitude. When the laser's wavelength is out of the Raman gain peak, the gain is not enough to amplify the laser seeds, and no more lasers wavelengths are formed. Since the Brillouin Stokes scattering generates counter-propagating waves, then observing the signal from the OSA, and counting the seed laser as the $0^{\text {th }}$ order Stokes laser, the odd order Stokes lasers (formed by Brillouin Stokes scattering) need an odd number of Rayleigh scattering reflections and the even order Stokes lasers need an even number of Rayleigh scattering reflections. Therefore, there is a difference of the odd and even order Stokes lasers [3].

The Raman gain of the DCF was observed using the experimental setup depicted in Fig. 1 (with the seed laser off) for different Raman pump powers and the maximum of the gain was observed to be at around $1555 \mathrm{~nm}$ (see fig. 2). The Raman pump power necessary to achieve the Rayleigh lasing threshold was 
measured to be $470 \mathrm{~mW}$ and for a Raman pump power of $500 \mathrm{~mW}$ random Rayleigh lasing peaks in the peak of the gain spectrum are observed.
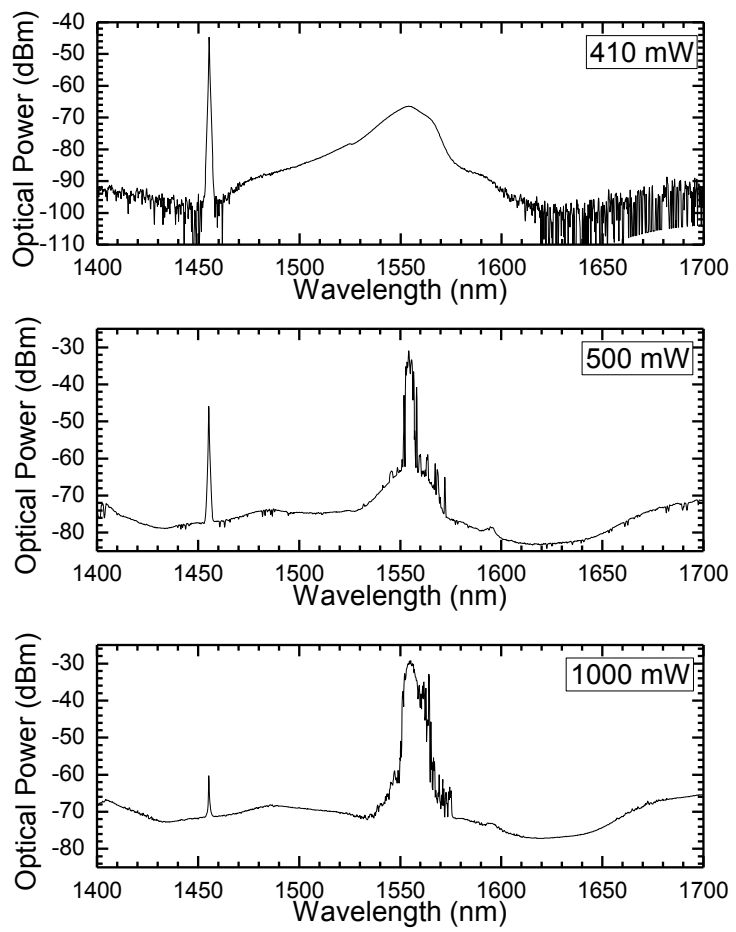

Fig. 2 Raman gain for different pump powers (seed laser off)

With the seed laser set to $10 \mathrm{dBm}$ at $1538 \mathrm{~nm}$, the gain spectrum for different Raman pump powers was observed (fig. 3). The Raman pump power necessary to achieve the Brillouin lasing threshold was measured to be $420 \mathrm{~mW}$. For a Raman pump power of $500 \mathrm{~mW}$ a flat-amplitude spectrum between 1538 $\mathrm{nm}$ and $1571 \mathrm{~nm}$ (centered in the maximum Raman gain peak), $20 \mathrm{~dB}$ above the optical power of nearby frequencies, was generated. Note that the resolution is not enough to see Rayleigh lasing peaks, but only the average energy of the spectrum. For higher pump powers, random Rayleigh lasing occurs at any wavelength in the comb. Therefore the Raman gain is higher in the earlier wavelengths of the comb, but lower power is left to amplify the later wavelengths of the comb. For higher pump powers, the average energy in the comb decreases with increasing wavelength and there is no sudden amplitude break at the end of the comb.

In fig. 4 the flat-amplitude spectrum of fig. 3 is observed for a) the whole spectrum (span $60 \mathrm{~nm}$ ) and b) magnified spectrum ( $\operatorname{span} 1 \mathrm{~nm}$ ) for different Raman pump powers with $10 \mathrm{dBm}$ seed laser at $1538 \mathrm{~nm}$. In fig. 4b, a Rayleigh lasing threshold (constant for all the pump powers) is observed at about $-57 \mathrm{dBm}$. For lower Raman pump powers, the Rayleigh lasing only occurs in the peaks of each laser, but for Raman pumps higher than $800 \mathrm{~mW}$, the minimum spectral power in the comb is above the Rayleigh lasing threshold, and therefore Rayleigh lasing can occur at any wavelength, generating a very unstable and noisy spectrum. Due to the random properties of the Rayleigh lasing the maximum gain in each peak is unstable, which generates a non-flat amplitude. 
For increasing Raman pump powers, the maximum (i.e. the average of the Rayleigh lasing peaks of each laser) and minimum power of the optical spectrum are higher. However, the minimum changes by approximately $9 \mathrm{~dB}$ (having an average of $-64,5 \mathrm{dBm}$ for $500 \mathrm{~mW}$ ) and the maximum changes by approximately $3 \mathrm{~dB}$ (having an average of $-46 \mathrm{dBm}$ for $500 \mathrm{~mW}$ ) between $500 \mathrm{~mW}$ and $1000 \mathrm{~mW}$ (fig. 4b). Therefore, the highest optical signal-to-noise ratio and stability and the lowest noise are achieved with lower Raman pump powers, immediately over the Raman pump power necessary to achieve the Brillouin lasing threshold. The difference between the form of wider (with higher amplitude average) and narrower Brillouin Stokes peaks can also be observed. The spacing between peaks was observed to be $0,08 \mathrm{~nm}$ and is constant for all the measurements in the work.

The last wavelength in the comb was observed to be higher for higher pump powers (fig. $4 \mathrm{a}$ ): $1571.5 \mathrm{~nm}$ for $500 \mathrm{~mW}$ (419 lasers) and $1572.5 \mathrm{~nm}$ (431 lasers) for $1000 \mathrm{~mW}$. Note that the resolution of fig. 4a is not enough to see the Rayleigh lasing peaks, but only the average of the laser peak (at approximately -55 $\mathrm{dBm}$ for $500 \mathrm{~mW}$ and $-46 \mathrm{dBm}$ for $1000 \mathrm{~mW}$ ). The Raman pump power necessary to achieve the Brillouin lasing threshold was measured to be $420 \mathrm{~mW}$.
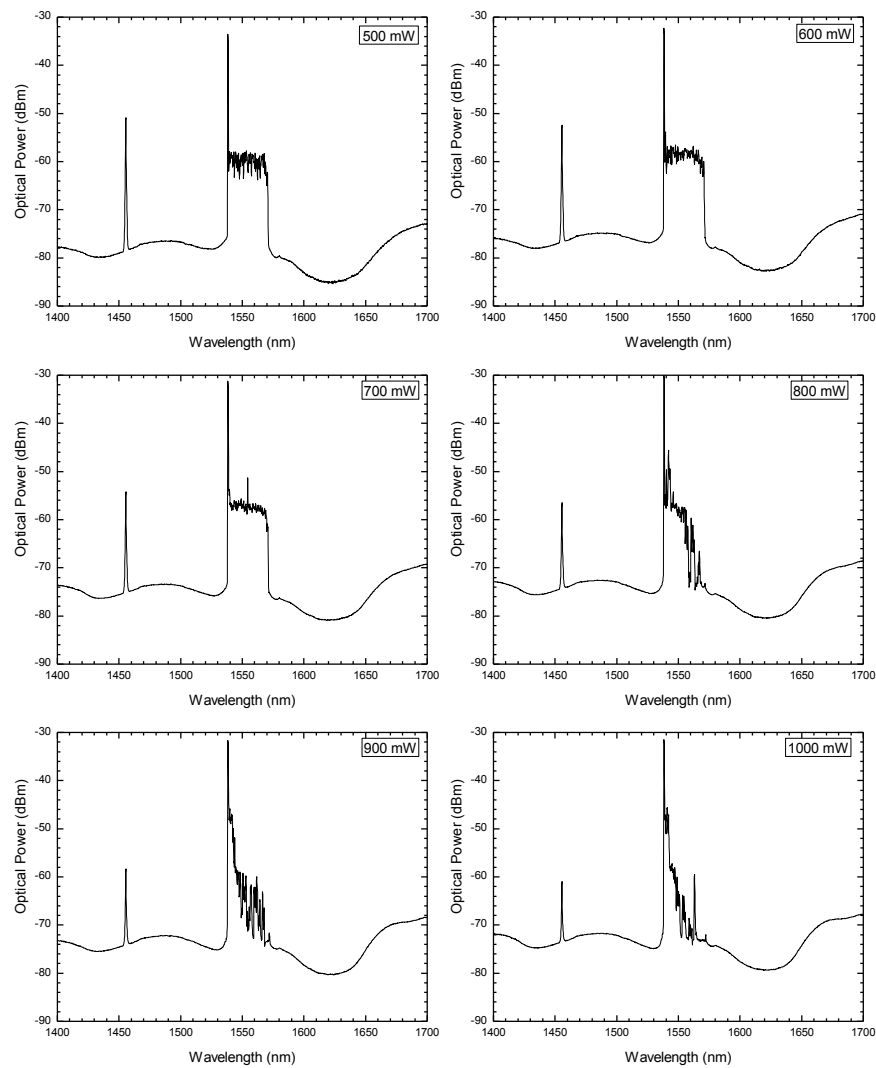

Fig. 3 Raman-Brillouin spectrum for different pump powers, with $10 \mathrm{dBm}$ seed at $1538 \mathrm{~nm}$ 

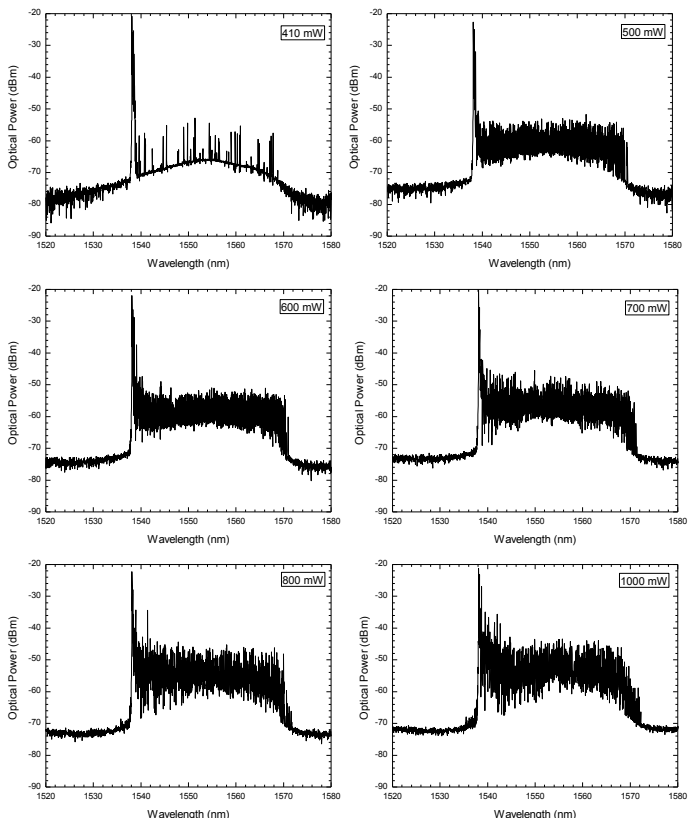

Fig. 4a Optical spectrum for different Raman pump powers, with $10 \mathrm{dBm}$ seed at $1538 \mathrm{~nm}(\operatorname{span} 60 \mathrm{~nm})$.
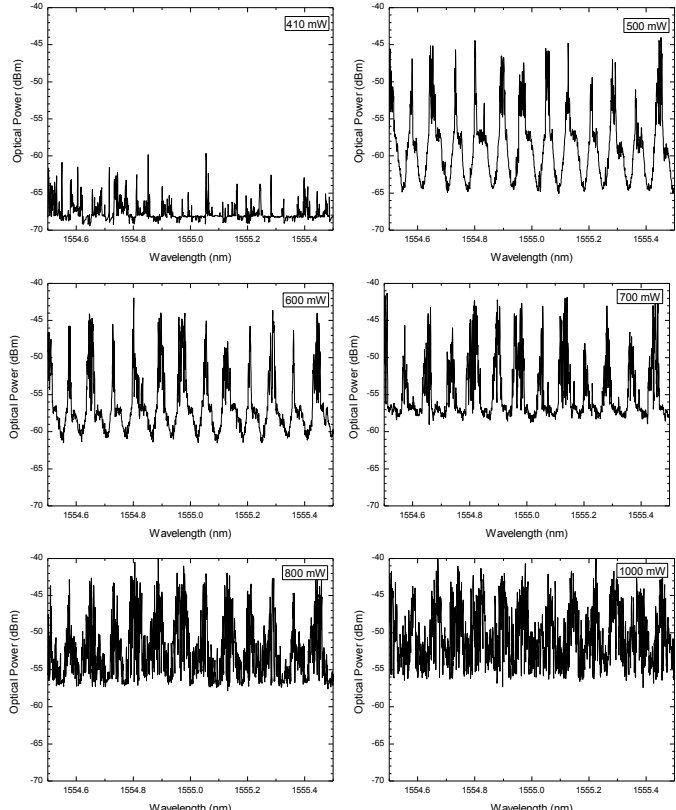

Fig. 4b Optical spectrum for different Raman pump powers, with $10 \mathrm{dBm}$ seed at $1538 \mathrm{~nm}$ (span $1 \mathrm{~nm}$ ).

In fig. 5 the flat-amplitude spectrum for a) the whole spectrum (span $60 \mathrm{~nm}$ ) and b) magnified spectrum (span $1 \mathrm{~nm}$ ) for different seed laser powers at $1538 \mathrm{~nm}$, with $500 \mathrm{~mW}$ of Raman pump power is observed. The seed laser power necessary to achieve the Brillouin cascaded threshold power was observed to be $2.5 \mathrm{dBm}$. Once the seed laser has enough power to start the process the spectrum remains almost constant for different powers. This happens because the power due to the Raman gain is much higher than the changes on the power of the seed laser.
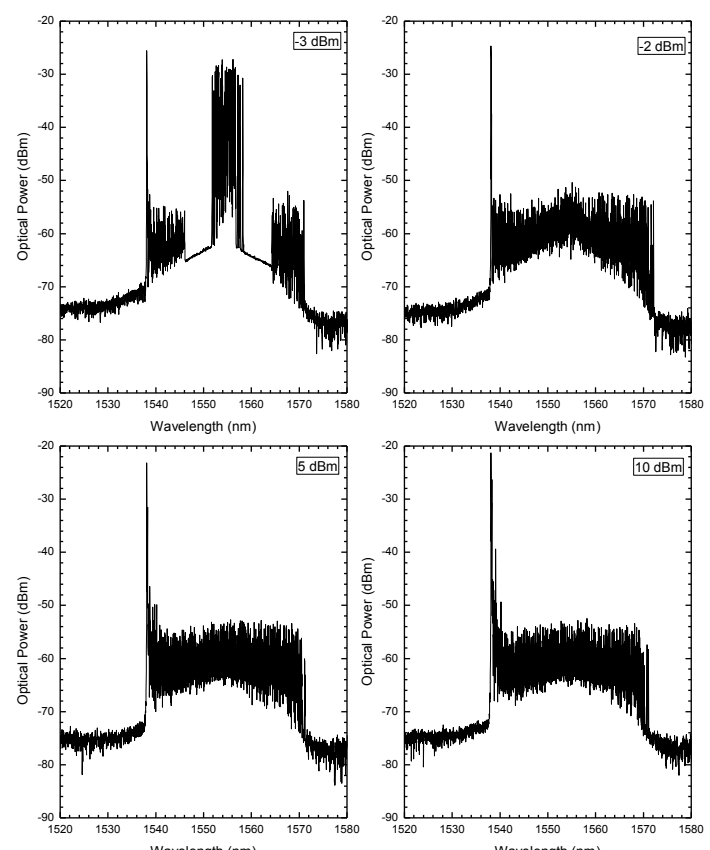

Fig. 5a Optical spectrum for different seed laser powers at $1538 \mathrm{~nm}$ with a Raman pump power of $500 \mathrm{~mW}$ (span $60 \mathrm{~nm})$.
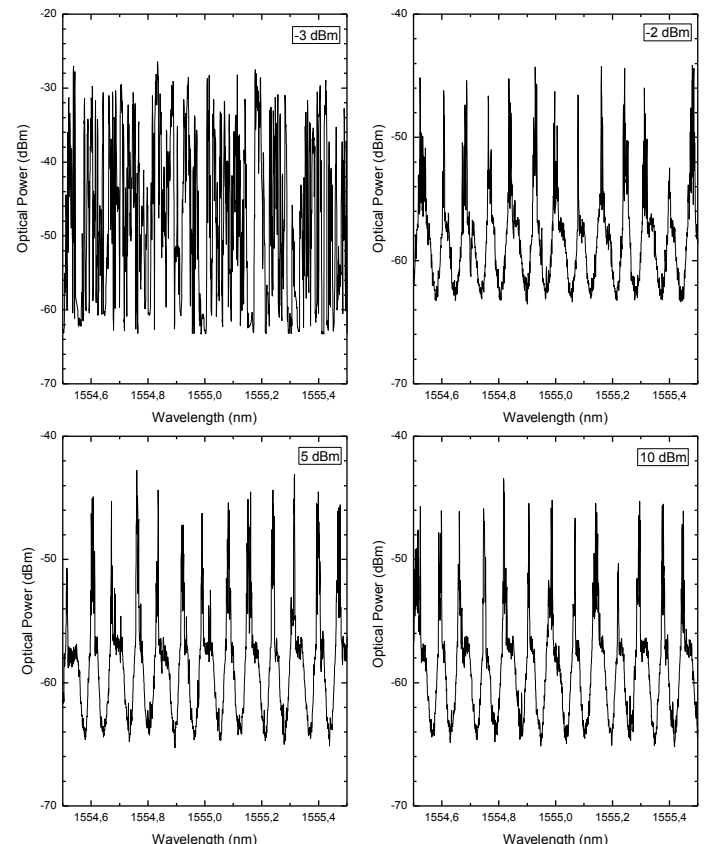

Fig. 5b Optical spectrum for different seed laser powers at $1538 \mathrm{~nm}$ with a Raman pump power of $500 \mathrm{~mW}$ (span $1 \mathrm{~nm})$. 
In fig. 6 the flat-amplitude spectrum for a) the whole spectrum (span $60 \mathrm{~nm}$ ) and b) magnified spectrum (span $1 \mathrm{~nm}$ ) for different seed laser wavelengths with $10 \mathrm{dBm}$ and $500 \mathrm{~mW}$ of Raman pump power is observed. Since the Raman gain is rapidly decreasing in the region of $1538 \mathrm{~nm}$, the spectrum is very sensible to these changes. For higher (closer to the maximum Raman gain peak) seed wavelengths there are less laser peaks without Rayleigh lasing amplification and therefore the spectrum amplitude is more uniform (fig. 6a). Also, the spectrum was also observed to be more stable and the average power of the spectrum was higher. However, the maximum power of the Rayleigh lasing peaks and the minimum of the optical power of the spectrum in the maximum of the Raman gain (fig. 6b) were approximately constant for the tested seed wavelengths.

The last wavelength of the comb (and therefore the bandwidth) was observed to be lower for higher seed wavelengths. For a seed laser wavelength below $1533 \mathrm{~nm}$, the Raman gain is not enough to start the Brillouin cascaded process.
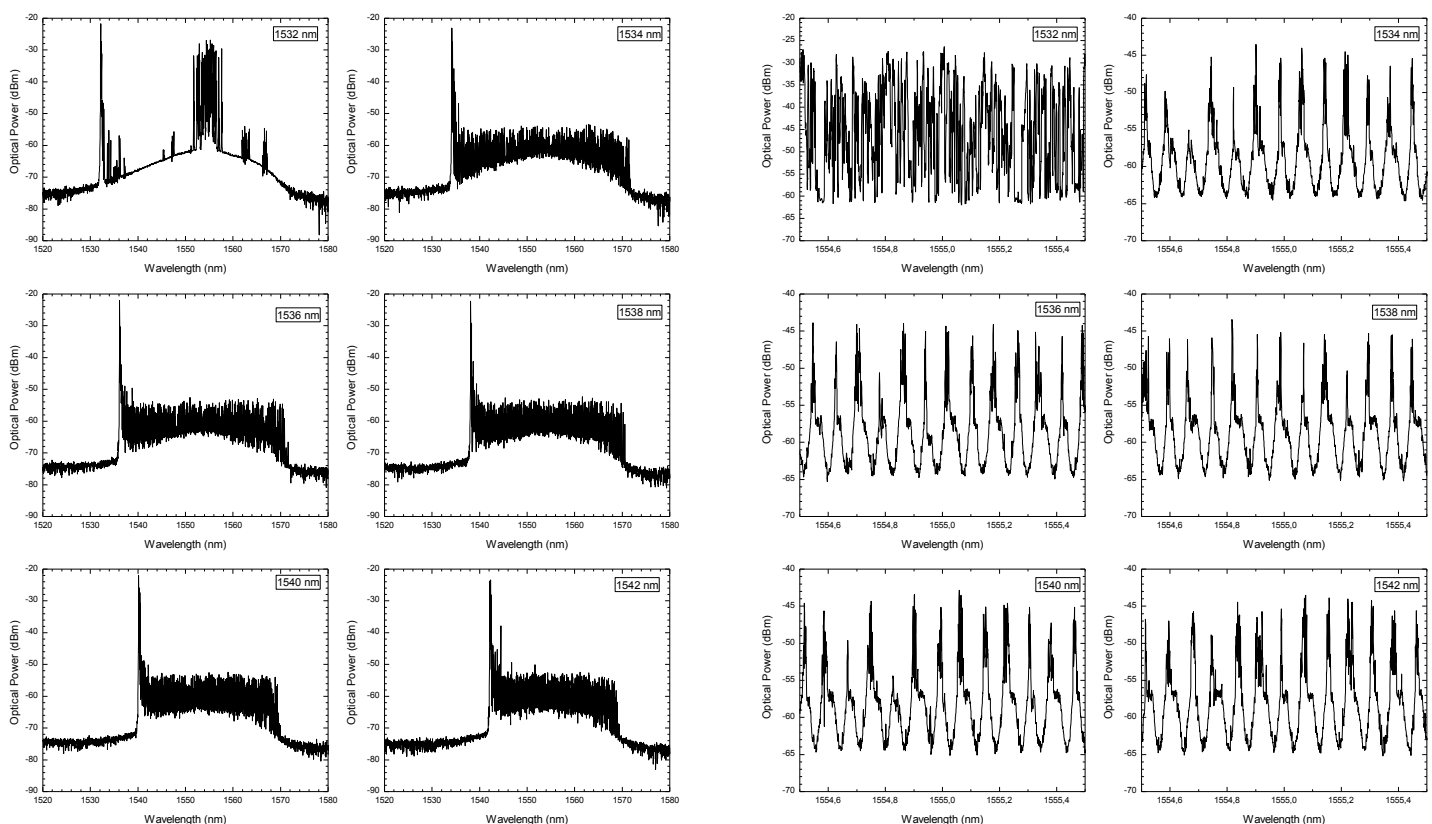

Fig. 6a Optical spectrum for different seed laser wavelengths with $10 \mathrm{dBm}$ and $500 \mathrm{~mW}$ of Raman pump power (span $60 \mathrm{~nm}$ ).
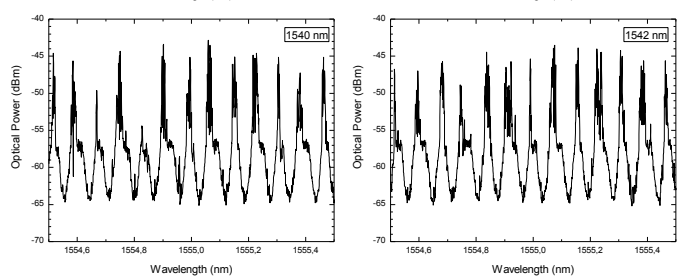

Fig. 6b Optical spectrum for different seed laser wavelengths with $10 \mathrm{dBm}$ and $500 \mathrm{~mW}$ of Raman pump power (span $1 \mathrm{~nm})$.

\section{CONCLUSION}

For lower Raman pump powers the spectrum was more stable, less noisy and the optical signal-to-noise ratio of the lasers was observed to be higher. The power of the seed laser did not affect the spectrum once it had passed the necessary power to achieve the threshold power. For lower wavelengths of the seed laser, the spectrum was more uniform and had a higher average optical power. However, the minimum and maximum optical power of the spectrum close to the Raman gain peak was almost constant. The last wavelength of the comb was observed to increase with higher Raman pump power and lower seed wavelength. A difference between the form of wider and narrower Brillouin Stokes peaks was observed and the spacing between consecutive peaks was measured to be $0.08 \mathrm{~nm}$. 


\section{REFERENCES}

[1] H. Takara, T. Ohara, K. Mori, K. -I. Sato, E. Yamada, Y. Inoue, T, Shibata, M. Abe, T. Marioka, and K. Sato, "More than 1000 channel optical frequency chain generation from single supercontinuum source with 12.5 GHz channel spacing”, Electron. Lett. 36, 2089-2090 (2000).

[2] J. Chow, G. Town, B. Eggleton, M. Ibsen, K. Sugden, and I. Bennion, "Multiwavelength generation in an erbium-fiber laser using in-fiber comb filters", IEEE Photon. Technol. Lett, 8, 60-62 (1996).

[3] K. D. Park, B. Min, P. Kim, N. Park, J. H. Lee, and J. S. Chang, "Dynamics of cascaded Brillouin-Rayleigh scattering in a distributed fiber Raman amplifier”, Opt. Lett. 27, 155-157 (2002).

[4] G. J. Cowle and D. Y. Stepanov, "Multiple wavelength generation with Brillouin/Erbium fibre lasers", IEEE Photon. Technol. Lett. 8, 1465-1467 (1996).

[5] B. Min, P. Kim, and N. Park, "Flat amplitude equal spacing 798 channel Rayleigh assisted Brillouin/Raman multiwavelength comb generation in dispersion compensating fiber", IEEE Photon. Technol. Lett. 13, 1352-1354 (2001).

[6] A.K. Zamzuri, M.I. Md. Ali, A. Ahmad, R. Mohamad, and M.A. Mahdi, "Brillouin-Raman comb fiber laser with cooperative Rayleigh scattering in a linear cavity", Opt. Lett. 31, 918-920 (2006).

[7] K. D. Park, H. Ryu, W. K. Lee, S. K. Kim, H. S. Moon, and H. S. Suh, "Threshold features of a Brillouin Stokes comb generated in a distributed fiber Raman amplifier”, Opt. Lett. 28, 13111313 (2003).

[8] A. K. Zamzuri, M. A. Mahdi, A. Ahmad, M. I. Md Ali, and M. H. Al-Mansoori, "Flat amplitude multiwavelength Brillouin-Raman comb fiber laser in Rayleigh-scattering enhanced linear cavity", Opt. Express., 15(6):3000-5 (2007). 